\title{
Direct Measurements of the Ionization Potential Depression in a Dense Plasma
}

\author{
O. Ciricosta, ${ }^{1}$ S. M. Vinko, ${ }^{1}$ H.-K. Chung, ${ }^{2}$ B.-I. Cho, ${ }^{3}$ C. R. D. Brown, ${ }^{4}$ T. Burian, ${ }^{5}$ J. Chalupský,,${ }^{5}$ K. Engelhorn, ${ }^{3}$ \\ R. W. Falcone, ${ }^{3,6}$ C. Graves, ${ }^{7}$ V. Hájková, ${ }^{5}$ A. Higginbotham, ${ }^{1}$ L. Juha, ${ }^{5}$ J. Krzywinski, ${ }^{7}$ H. J. Lee, ${ }^{7}$ M. Messerschmidt, ${ }^{7}$ \\ C. D. Murphy, ${ }_{1}^{1}$ Y. Ping, ${ }^{8}$ D. S. Rackstraw, ${ }^{1}$ A. Scherz, ${ }^{7}$ W. Schlotter, ${ }^{7}$ S. Toleikis, ${ }^{9}$ J. J. Turner, ${ }^{7}$ L. Vysin, ${ }^{5}$ T. Wang ${ }^{7}$ \\ B. Wu, ${ }^{7}$ U. Zastrau, ${ }^{10}$ D. Zhu, ${ }^{7}$ R. W. Lee, ${ }^{7}$ P. Heimann, ${ }^{3}$ B. Nagler, ${ }^{7}$ and J. S. Wark ${ }^{1, *}$ \\ ${ }^{1}$ Department of Physics, Clarendon Laboratory, University of Oxford, Parks Road, Oxford OX1 3PU, United Kingdom \\ ${ }^{2}$ Atomic and Molecular Data Unit, Nuclear Data Section, IAEA, P.O. Box 100, A-1400, Vienna, Austria \\ ${ }^{3}$ Lawrence Berkeley National Laboratory, 1 Cyclotron Road, California 94720, USA \\ ${ }^{4}$ Plasma Physics Department, AWE Aldermaston, Reading, United Kingdom \\ ${ }^{5}$ Institute of Physics ASCR, Na Slovance 2, 18221 Prague 8, Czech Republic \\ ${ }^{6}$ Department of Physics, University of California, Berkeley, California 94720, USA \\ ${ }^{7}$ SLAC National Accelerator Laboratory, 2575 Sand Hill Road, Menlo Park, California 94025, USA \\ ${ }^{8}$ Lawrence Livermore National Laboratory, 7000 East Avenue, Livermore, California 94550, USA \\ ${ }^{9}$ Deutsches-Elektronensynchrotron DESY, Notkestrasse 85, 22603 Hamburg, Germany \\ ${ }^{10}$ IOQ, Friedrich-Schiller-Universität Jena, Max-Wien-Platz 1, 07743 Jena, Germany
}

(Received 30 March 2012; published 6 August 2012)

\begin{abstract}
We have used the Linac Coherent Light Source to generate solid-density aluminum plasmas at temperatures of up to $180 \mathrm{eV}$. By varying the photon energy of the $\mathrm{x}$ rays that both create and probe the plasma, and observing the $K$ - $\alpha$ fluorescence, we can directly measure the position of the $K$ edge of the highly charged ions within the system. The results are found to disagree with the predictions of the extensively used Stewart-Pyatt model, but are consistent with the earlier model of Ecker and Kröll, which predicts significantly greater depression of the ionization potential.
\end{abstract}

DOI: 10.1103/PhysRevLett.109.065002

PACS numbers: 52.20.-j, 52.25.Os, 52.70.La

In a plasma the distribution of the electrostatic potential surrounding an ion is influenced by the free electrons and neighboring ions. As a result, the threshold energy required to further ionize a given ion by exciting one of the bound electrons to the continuum is lowered from that of the equivalent isolated ion [1-3], a phenomenon known as ionization potential depression (IPD). For a dense plasma, the IPD can significantly alter the ionization balance and limit the number of accessible bound states (pressure ionization), shifting the charge state distribution (CSD) in the direction of increased ionization [4,5]. This strongly affects the thermodynamic properties of the system, including its equation of state and opacity, and as such the IPD is of fundamental importance for astrophysics and cosmology [6], planetary science [7], and inertial confinement fusion research $[8,9]$.

At low plasma densities and high temperatures, the IPD is determined by the charge screening effects described within the Debye-Hückel (DH) theory [10]. However, at high electron number densities the DH model breaks down, as the characteristic screening length (the Debye length $\left.\lambda_{D}=\sqrt{\varepsilon_{0} k_{\mathrm{B}} T / n_{e} e^{2}}\right)$ becomes smaller than the interparticle distance. Over the past half a century several models have been proposed for the IPD within such dense systems. The simplest approach is the ion-sphere (IS) average atom model, where one defines a radius, $R_{0}$, of a neutral sphere containing an ion with charge $z^{*}$, i.e., $4 \pi R_{0}^{3} / 3=z^{*} / n_{e}=$ $1 / n_{i}$, with a resultant IPD of $\Delta I=C_{\mathrm{IS}} z^{*} e^{2} /\left(4 \pi \varepsilon_{0} R_{0}\right)$, where the value of the constant is taken to be $C_{\text {IS }}=9 / 5$ by Zimmerman and More [3]. The most widely used model for detailed atomic configurations calculations that interpolates between the DH and IS limits is the one put forward by Stewart and Pyatt (SP) [2]: it is the model used in CRETIN [11], FLYCHK [12], and LASNEX-DCA [13] codes (amongst many others), which have been used extensively over the past few decades to simulate hot-dense plasmas. In the high density limit, the SP model yields an IS form of the IPD, where the average value $z^{*}$ is replaced by the charge $z$ of the ion resulting from the ionization process (i.e., $z=1$ for the neutral atom), and $C_{\mathrm{IS}}$ is $3 / 2$. However, prior to the SP model, Ecker and Kröll (EK) [1] posited that, rather than $R_{0}$, the relevant length for determining the IPD at high densities was the average distance between all free particles, i.e., the radius $r_{\mathrm{EK}}$ defined by $r_{\mathrm{EK}}^{3}=$ $3 /\left(4 \pi\left[n_{e}+n_{i}\right]\right)$, which for a highly ionized system is approximately the mean separation of the free electrons (for low densities the same DH limit as in the SP model is found). The EK model then assumes an IPD of $\Delta I=$ $C_{\mathrm{EK}} z e^{2} /\left(4 \pi \varepsilon_{0} r_{\mathrm{EK}}\right)$, where $C_{\mathrm{EK}} \approx 1$, and $z$ is defined in the same way as in the SP model. For highly ionized systems the EK model predicts a far higher IPD than the SP model, with an additional scaling of $z^{1 / 3}$.

Despite its fundamental importance, experimental data on the IPD within dense plasmas remain elusive. The few attempts made to investigate this phenomenon rely on spectroscopy, measuring continuum edge shifts, and 
observing transitions involving the highest energy bound states surviving pressure ionization [14-16]. However this approach is suspect: not only is the energy of the last bound state not the same as the lowest energy of the continuum, it is also difficult to distinguish between the real continuum edge and the point at which the spectroscopic series appears to merge into a continuum (the so-called Inglis-Teller limit [17]), which is strongly dependent on line broadening. Furthermore, there is no systematic study of the IPD in a highly charged dense system due to the difficulties of creating samples that are dense and ionized at the same time, and with an accurately known density. Thus our current understanding of one of the most important factors that influences the fundamental properties of dense plasma systems is woefully incomplete.

It is in this context that we report on a technique that overcomes all of the above problems, and affords the means to measure the IPD within a dense plasma in a situation where the IPD-relevant radii can be easily determined. Importantly, we find that our experimental results cannot be reproduced by the SP approach, but are consistent with the earlier EK model which predicts substantially larger values of the IPD.

Our technique relies on irradiating a solid foil with the focused output of an x-ray free-electron laser. The salient features of the setup of the experiment, performed on the soft x-ray (SXR) instrument of the Linac Coherent Light Source (LCLS) [18], have been described in detail elsewhere [19]. Targets comprising $1 \mu \mathrm{m}$ thick $\mathrm{Al}$ foils were irradiated by 80 fs pulses of $x$ rays, with photon energies in the range 1540-1830 eV (around and above the $K$ edge of cold Al) at peak intensities exceeding $10^{17} \mathrm{~W} \mathrm{~cm}^{-2}$. At these irradiances the electrons within the target are heated to temperatures between 70 and $180 \mathrm{eV}$, depending on the LCLS photon energy, as indicated by our simulations (see below). The primary absorption mechanism is direct photoionization, resulting in the creation of $K$-shell holes. The majority of those $K$-shell holes are filled as a result of the $K L L$ Auger decay, with approximately $3 \%$ of them filled by radiative decay from the $L$ to $K$ shell, resulting in an $\mathrm{x}$-ray fluorescence spectrum recorded by means of a flat crystal Bragg spectrometer. As the electrons within the $1 \mu \mathrm{m}$ foil are heated within $80 \mathrm{fs}$, ion motion is negligible (even if electron-ion energy transfer were instantaneous, a $200 \mathrm{eV} \mathrm{Al}$ ion only moves $5 \mathrm{~nm}$ in $80 \mathrm{fs}$, thus the heating is almost perfectly isochoric). Since the $K$-shell fluorescence only occurs while the target is irradiated by LCLS (the temperature is too low for thermal ionization of the $K$ shell, and the $K$-shell core hole lifetime is significantly shorter than the LCLS pulselength), emission occurs from ions within a plasma where the ion density $n_{i}$ is exactly known, being equal to the solid-density value, and thus the electron density is trivially related to the amount of ionization.

In Fig. 1 we plot a subset of the recorded spectra for six different photon energies of the $\mathrm{x}$-ray laser pump. The figure

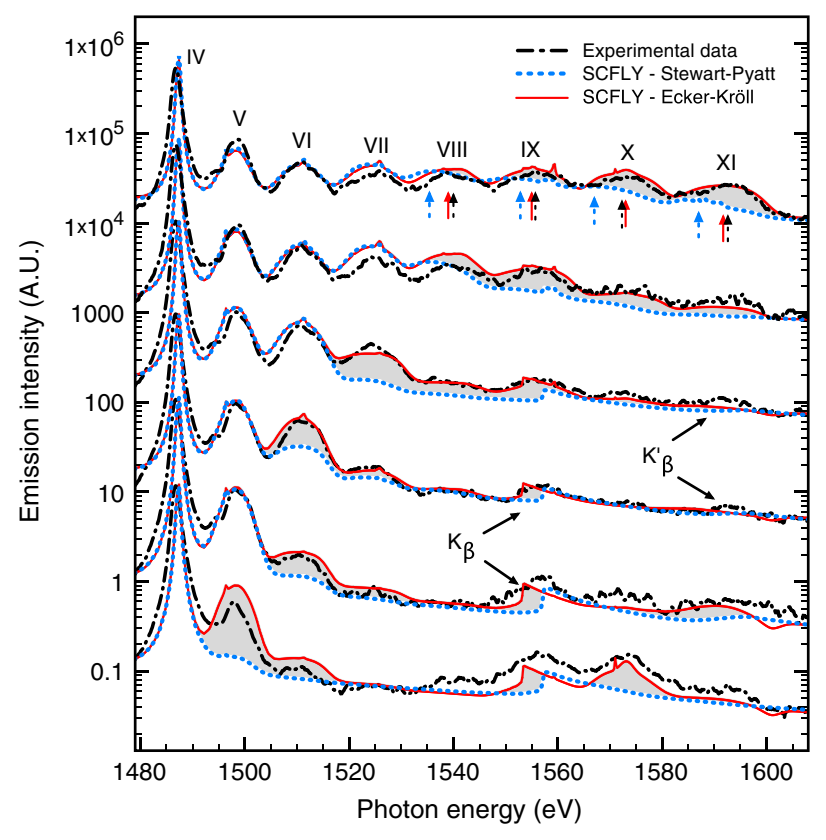

FIG. 1 (color online). Experimentally recorded $K$ - $\alpha$ emission spectra from hot solid-density aluminum. SCFLY simulations with different IPD models are compared with the experimental data for a subset of the x-ray laser photon energies. From the bottom, the spectra corresponding to $1580,1600,1630,1650,1720$ and $1830 \mathrm{eV}$ pump photon energies are shown. The spectra have been artificially displaced in intensity for clarity, and a bremsstrahlung component (at the maximum temperature provided by the simulations) has been added to the calculated spectra. The grey shading indicates the difference between the calculations using different IPD models.

shows the series of $K$ - $\alpha$ emission lines corresponding to transitions of $L$ electrons filling $K$-shell holes, created by the LCLS photons, in ions with a different number of $L$-shell holes, labeled by their charge state (recall that cold solid density Al already has 3 free electrons, corresponding to a pressure-ionized $M$ shell). Importantly, apart from when a resonance occurs, a $K$-shell hole in a specific ion can only be created by the monochromatic $x$-ray laser if the photon energy exceeds the $K$ absorption edge of that ion within the plasma, while, as we have shown in [19], the CSD in the system is only weakly dependent on the wavelength used; thus, the appearance and intensity of the $K$ - $\alpha$ emission peak of the highest absorbing charge state as a function of LCLS photon energy is a sensitive indicator of the energy of the $K$ edge of that ion stage. Although resonant $K-L$ shell transitions can occur, they are well separated in energy with respect to the $K$-edge values. Thus by tuning the photon energy of LCLS, and observing the appearance of the $K$ - $\alpha$ peaks from the ions we can determine the position of the $K$ edges, and thus the IPD, for the plasma parameters determined by the pumping conditions. Importantly, the diagnostic indicating ionization-i.e., the creation of the $K$-shell hole by promoting an electron into the continuumis the subsequent radiative transition of an electron in an 
$L$ shell into the vacant $K$-shell state. That is to say that we are not reliant on observing emission from states very close to the continuum, that are subject to significant line broadening, in order to determine whether or not ionization has occurred.

We model our experimental results with atomic kinetics simulations using the collisional-radiative superconfiguration code SCFLY [20]. The code is specifically tailored to $\mathrm{x}$-ray laser related problems, and has previously been tested in the noncollisional regime [21]. It uses a rate equation formalism to calculate the time evolution of the atomic populations, taking into account the effects of the IPD for the different ionization stages, and provides as an output the time resolved temperature, density, CSD, opacity and emission spectra. We have incorporated the SP (the full analytical model as in Ref. [2]) and EK models of IPD within the code. The spatial variation in intensity across the approximately $9.1 \pm 0.8 \mu \mathrm{m}^{2}$ area of the LCLS focal spot was taken into account, by appropriately summing and weighting simulations at different intensities, with the spatial profile of the focal spot determined by laser imprints in $\mathrm{PbWO}_{4}$ taken during the experiment [22].

A comparison between the experimental and simulated spectra is shown in Fig. 1. It can be observed that the SP calculations do not correctly reproduce the dependence of the $K$ - $\alpha$ spectra on the LCLS photon energy, while the EK model provides excellent agreement in reproducing the appearance, intensity, and positions of the various $K-\alpha$ peaks. For example, the SP model cannot predict the appearance of the V $K$ - $\alpha$ line at an LCLS photon energy of $1580 \mathrm{eV}$, the VI line (at $1630 \mathrm{eV}$ ), or the lines X and XI (at $1830 \mathrm{eV}$ ). In contrast the EK model provides a striking agreement with the experimental $K-\alpha$ spectra.

In Fig. 2 we plot the experimentally detected and calculated $K$ edges, and the corresponding IPD values, for the first five charge states. The experimental $K$ edges are determined by the appearance of the peaks in the spectra as a function of the LCLS photon energy, while the calculated ones are given by simulations performed at photon energies given by the experimental edges, taking the edge value at the time where the corresponding line emission is maximum. The last three charge states are not shown because determining the $K$-edge position for these lines is complicated by the presence of the overlapping $K-\beta$ series. The calculated energies of the shells of the free ions are also shown, demonstrating that only the $K$ and $L$ shell states are bound at these densities within the EK model. The experimental IPD values shown on the right part of the figure are determined by the difference between the atomic and detected edge values, while the solid colored parts of the histograms' bars for the simulated values illustrate the variation of the IPD as the electron density increases during the evolution of the system. The success of the EK calculations compared with those employing the SP model is clear.

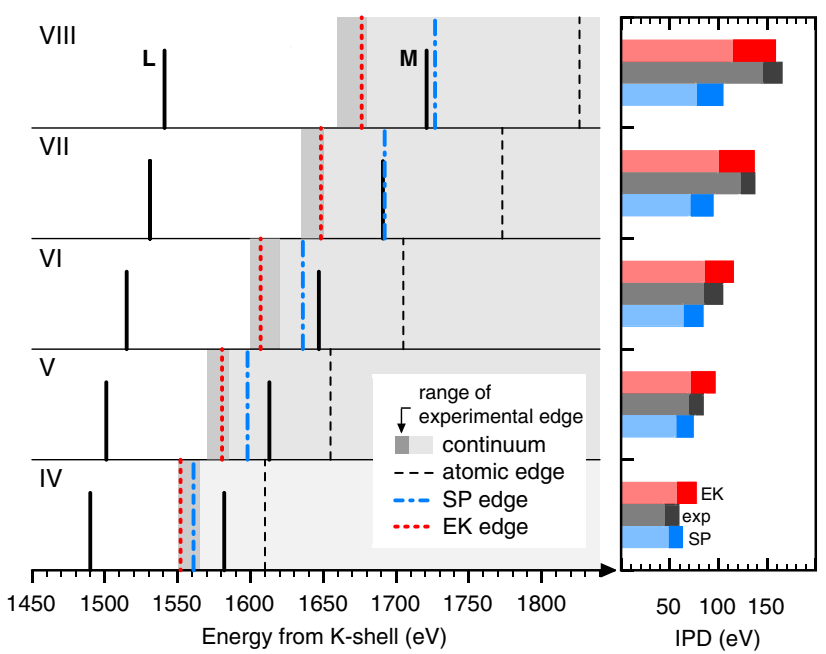

FIG. 2 (color online). Left-The grey region shows the continuum for different charge states, as determined from the experimental spectra in Fig. 1, with the dark grey region corresponding to the observed range of the $K$ edge. The values given by the SP and EK calculations correspond to the edges calculated at the time of maximum emission for each associated $K-\alpha$ line. The calculated energy to pump a $K-L$ and $K-M$ transition (for the same number of $L$ shell electrons in the final state) is also indicated. Right-IPD values; the darker colored zones of the histogram correspond to the IPD variation detected, for the experimental bars, and to the total IPD variation during the system evolution, for the simulated ones.

The IPD has an influence not only on the number of $K$ - $\alpha$ peaks observed, but also on the energy at which they are emitted. For highly charged $\mathrm{Al}$ ions, as can be seen in Fig. 2 for the charge states VII and VIII, the SP model predicts a far smaller IPD, so that the lowest energy of the continuum is such that the $M$ shell rebinds, resulting in greater screening of the $L$-shell electrons, and a shift in the $K$ - $\alpha$ energy [23]. In contrast, the larger IPD predicted by EK means that the $M$ shell is pressure ionized for all the ions (as in the cold solid). The effect of this difference can be seen again in Fig. 1, where the arrows under the spectrum corresponding to $1830 \mathrm{eV}$ pumping show that the simulations using the SP model predict the wrong position for the VII-XI $K-\alpha$ lines, while the EK model agrees with experiment; i.e., the data demonstrate that the $M$ shell is indeed pressure ionized. This effect explains the discrepancies in the energies of the $K-\alpha$ lines in the simulations presented in [19], which used a modified version of the SP model.

As additional evidence, in Fig. 3 we plot the spectra showing the secondary $K$ - $\alpha$ series, corresponding to the emission from atoms with a doubly ionized $K$ shell [24]. Since the energy threshold values for ionizing a second electron from the $K$ shell are higher than the $K$ edges for the main satellite series, the progressive appearance of these lines occurs at higher pump photon energies. The four lines shown are emitted from the same ion stages responsible for the emission of the lines V-VIII in the main series, so the 


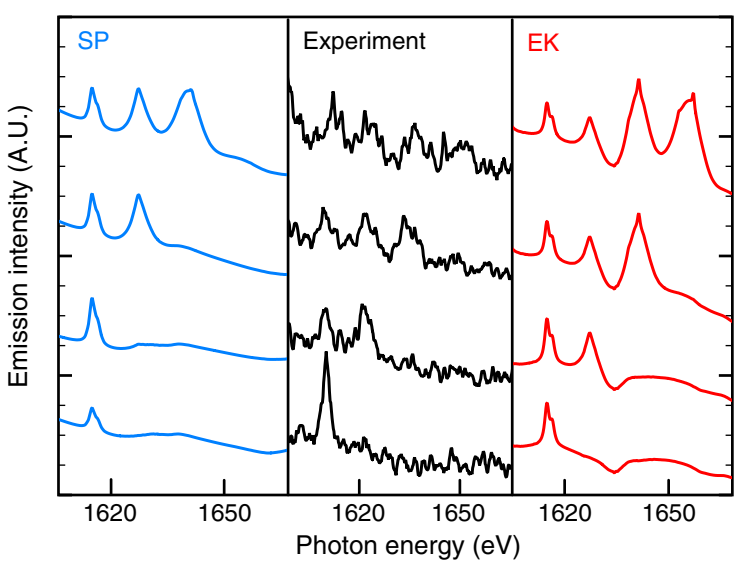

FIG. 3 (color online). Observation of the $K$-shell fluorescence corresponding to initial states with double core holes, along with SCFLY simulations using the SP and EK models. From the bottom, the spectra correspond to $1720,1750,1780,1805 \mathrm{eV}$ pump photon energies.

IPD determining their $K$-edge level will follow the same $z$ dependence, but with a free-electron density determined by different pumping conditions. When comparing the experimental trend with simulations, again the SP model is found not to be able to reproduce the correct $K$-edge values, while the EK model correctly predicts their appearance.

In conclusion we have presented an experimental study that provides detailed information on the IPD within a dense plasma. The new generation of intense short-pulse tunable $\mathrm{x}$-ray free-electron lasers allows the detection of the $K$ edges of the ion stages as the system is driven into the hot-dense regime by photoabsorption, and the observation of the various charge states from fluorescence provides a detection method that is not susceptible to the problems of line broadening of transitions from high-lying states. We find that the SP model cannot reproduce the values of the IPD found within this strongly coupled system, while the earlier EK model, predicting higher IPD values, gives excellent agreement with the experimental data. The reasons underlying the success of the EK over the SP model are far from clear: both the SP and the EK models are simple, semiclassical models, ultimately both unlikely to capture fully the complex physics of atomic systems embedded within dense plasma environments over wide ranges of plasma conditions and charge states. However, given the wide use of the SP model in astrophysical and inertial confinement fusion plasma simulations, and the consequent implications for errors in the predicted degree of ionization in such systems and associated consequences for the equation of state and opacity, we posit that this work which clearly demonstrates IPDs far greater than the SP model predicts, yet consistent with the work of EK, ought to spur further efforts to produce more sophisticated self-consistent models of the IPD within dense plasmas.

Portions of this research were carried out on the SXR instrument at the LCLS, a division of SLAC National
Accelerator Laboratory and an Office of Science user facility operated by Stanford University for the US Department of Energy. O. C., S. M. V., D. S. R., and J. S. W. thank the UK EPSRC for funding (EP/F020449/1 and EP/ H035877/1). T.B., J.C., V.H., L.J. and L. V. appreciate funding Grants No. P208/10/2302, No. P205/11/0571, No. P108/11/1312, No. ME10046, and No. IAAX00100903. K.E., R.W.F., P. A.H. acknowledge US DOE Basic Energy Science Contract No. DE-AC0376SF00098 and SSAA program Contract No. DE-FG5206NA26212. U.Z. acknowledges funding by the German Ministry for Education and Research (BMBF) via FSP 301 and the Volkswagen Foundation via a PPE Fellowship.

*Corresponding author. justin.wark@physics.ox.ac.uk

[1] G. Ecker and W. Kröll, Phys. Fluids 6, 62 (1963).

[2] J. C. Stewart and K. D. Pyatt, Jr., Astrophys. J. 144, 1203 (1966).

[3] G. Zimmerman and R. More, J. Quant. Spectrosc. Radiat. Transfer 23, 517 (1980).

[4] D. D. Burgess and R. W. Lee, J. Phys. (Paris), Colloq. 43, C2-413 (1982).

[5] R. M. More, J. Quant. Spectrosc. Radiat. Transfer 27, 345 (1982).

[6] F. J. Rogers and C. A. Iglesias, Science 263, 50 (1994).

[7] T. Guillot, Science 286, 72 (1999).

[8] J. D. Lindl, P. Amendt, R. L. Berger, S. G. Glendinning, S. H. Glenzer, S. W. Haan, R. L. Kauffman, O. L. Landen, and L. J. Suter, Phys. Plasmas 11, 339 (2004).

[9] S. X. Hu, B. Militzer, V. N. Goncharov, and S. Skupsky, Phys. Rev. Lett. 104, 235003 (2010).

[10] H. R. Griem, Plasma Spectroscopy (McGraw-Hill, New York, 1964).

[11] H. A. Scott, J. Quant. Spectrosc. Radiat. Transfer 71, 689 (2001).

[12] H.-K. Chung, M. Chen, W. Morgan, Y. Ralchenko, and R. Lee, High Energy Density Phys. 1, 3 (2005).

[13] Y. T. Lee, J. Quant. Spectrosc. Radiat. Transfer 38, 131 (1987).

[14] D. K. Bradley, J. Kilkenny, S. J. Rose, and J. D. Hares, Phys. Rev. Lett. 59, 2995 (1987).

[15] D. Riley, O. Willi, S. J. Rose, and T. Afshar-Rad, Europhys. Lett. 10, 135 (1989).

[16] M. Nantel, G. Ma, S. Gu, C. Y. Côté, J. Itatani, and D. Umstadter, Phys. Rev. Lett. 80, 4442 (1998).

[17] D. R. Inglis and E. Teller, Astrophys. J. 90, 439 (1939).

[18] LCLS home page, http://lcls.slac.stanford.edu/.

[19] S. M. Vinko et al., Nature (London) 482, 59 (2012).

[20] H.-K. Chung, M. Chen, and R. Lee, High Energy Density Phys. 3, 57 (2007).

[21] O. Ciricosta, H.-K. Chung, R. W. Lee, and J. S. Wark, High Energy Density Phys. 7, 111 (2011).

[22] J. Chalupský et al., Opt. Express 18, 27836 (2010).

[23] J. J. MacFarlane, P. Wang, J. Bailey, T. A. Mehlhorn, R. J. Dukart, and R. C. Mancini, Phys. Rev. E 47, 2748 (1993).

[24] J. Auerhammer, H. Genz, A. Kumar, and A. Richter, Phys. Rev. A 38, 688 (1988). 